\title{
HARTMAN-MYCIELSKI FUNCTOR OF NON-METRIZABLE COMPACTA
}

\author{
Taras Radul AND Dušan Repovš
}

\begin{abstract}
We investigate some topological properties of a normal functor $H$ introduced earlier by Radul which is a certain functorial compactification of the HartmanMycielski construction $H M$. We show that $H$ is open and find the condition when $H X$ is an absolute retract homeomorphic to the Tychonov cube.
\end{abstract}

\section{Introduction}

The general theory of functors acting on the category $\mathcal{C}$ omp of compact Hausdorff spaces (compacta) and continuous mappings was founded by Shchepin [Sh2]. He described some elementary properties of such functors and defined the notion of the normal functor which has become very fruitful. The classes of all normal and weakly normal functors include many classical constructions: the hyperspace exp, the space of probability measures $P$, the superextension $\lambda$, the space of hyperspaces of inclusion $G$, and many other functors (cf. [FZ] and [TZ]).

Let $X$ be a space and $d$ an admissible metric on $X$ bounded by 1. By $H M(X)$ we shall denote the space of all maps from $[0,1)$ to the space $X$ such that $f \mid\left[t_{i}, t_{i+1}\right) \equiv$ const, for some $0=t_{0} \leq \cdots \leq t_{n}=1$, with respect to the following metric

$$
d_{H M}(f, g)=\int_{0}^{1} d(f(t), g(t)) d t, \quad f, g \in H M(X) .
$$

The construction of $H M(X)$ is known as the Hartman-Mycielski construction [HM].

For every $Z \in \mathcal{C}$ omp consider

$$
\begin{aligned}
& H M_{n}(Z)=\{f \in H M(Z) \mid \text { there exist } 0=t_{1}<\cdots<t_{n+1}=1 \\
&\text { with } \left.f \mid\left[t_{i}, t_{i+1}\right) \equiv z_{i} \in Z, i=1, \ldots, n\right\} .
\end{aligned}
$$

Let $\mathcal{U}$ be the unique uniformity of $Z$. For every $U \in \mathcal{U}$ and $\varepsilon>0$, let

$$
<\alpha, U, \varepsilon>=\left\{\beta \in H M_{n}(Z) \mid m\left\{t \in[0,1) \mid\left(\alpha(t), \beta\left(t^{\prime}\right)\right) \notin U\right\}<\varepsilon\right\} .
$$

The sets $\langle\alpha, U, \varepsilon\rangle$ form a base of a compact Hausdorff topology in $H M_{n} Z$. Given a map $f: X \rightarrow Y$ in $\mathcal{C}$ omp, define a map $H M_{n} X \rightarrow H M_{n} Y$ by the formula $H M_{n} F(\alpha)=f \circ \alpha$. Then $H M_{n}$ is a normal functor in $\mathcal{C}$ omp (cf. [TZ; 2.5.2]).

1991 Mathematics Subject Classification. 54B30, 57N20.

Key words and phrases. Hartman-Mycielski construction, absolute retract, Tykhonov cube, normal functor.

The research was supported by the Slovenian-Ukrainian grant SLO-UKR 06-07/04. 
For $X \in \mathcal{C} o m p$ we consider the space $H M X$ with the topology described above. In general, $H M X$ is not compact. Zarichnyi has asked if there exists a normal functor in $\mathcal{C} o m p$ which contains all functors $H M_{n}$ as subfunctors (see [TZ]). Such a functor $H$ was constructed in [Ra]. It was shown in [RR] that $H X$ is homeomorphic to the Hilbert cube for each non-degenerated metrizable compactum $X$.

We investigate some topological properties of the space $H X$ for non-metrizable compacta $X$. The main results of this paper are:

Theorem 1.1. Hf is open if and only if $f$ is an open map.

Theorem 1.2. $H X$ is an absolute retract if and only if $X$ is openly generated compactum of weight $\leq \omega_{1}$.

Theorem 1.3. $H X$ is homeomorphic to Tychonov cube if and only if $X$ is an openly generated $\chi$-homogeneous compactum of weight $\omega_{1}$.

\section{Construction of $H$ and its connection with the functor of probability measures $P$}

Let $X \in \mathcal{C}$ omp. By $C X$ we denote the Banach space of all continuous functions $\varphi: X \rightarrow \mathbb{R}$ with the usual sup-norm: $\|\varphi\|=\sup \{|\varphi(x)| \mid x \in X\}$. We denote the segment $[0,1]$ by $I$.

For $X \in \mathcal{C}$ omp let us define the uniformity of $H M X$. For each $\varphi \in C(X)$ and $a, b \in[0,1]$ with $a<b$ we define the function $\varphi_{(a, b)}: H M X \rightarrow \mathbb{R}$ by the following formula

$$
\varphi_{(a, b)}=\frac{1}{(b-a)} \int_{a}^{b} \varphi \circ \alpha(t) d t .
$$

Define

$$
S_{H M}(X)=\left\{\varphi_{(a, b)} \mid \varphi \in C(X) \text { and }(a, b) \subset[0,1)\right\} .
$$

For $\varphi_{1}, \ldots, \varphi_{n} \in S_{H M}(X)$ define a pseudometric $\rho_{\varphi_{1}, \ldots, \varphi_{n}}$ on $H M X$ by the formula

$$
\rho_{\varphi_{1}, \ldots, \varphi_{n}}(f, g)=\max \left\{\left|\varphi_{i}(f)-\varphi_{i}(g)\right| \mid i \in\{1, \ldots, n\}\right\},
$$

where $f, g \in H M X$. The family of pseudometrics

$$
\mathcal{P}=\left\{\rho_{\varphi_{1}, \ldots, \varphi_{n}} \mid n \in \mathbb{N}, \text { where } \varphi_{1}, \ldots, \varphi_{n} \in S_{H M}(X)\right\},
$$

defines a totally bounded uniformity $\mathcal{U}_{H M X}$ of $H M X$ (see [Ra]).

For each compactum $X$ we consider the uniform space $\left(H X, \mathcal{U}_{H X}\right)$ which is the completion of $\left(H M X, \mathcal{U}_{H M X}\right)$ and the topological space $H X$ with the topology induced by the uniformity $\mathcal{U}_{H X}$. Since $\mathcal{U}_{H M X}$ is totally bounded, the space $H X$ is compact.

Let $f: X \rightarrow Y$ be a continuous map. Define the map $H M f: H M X \rightarrow H M Y$ by the formula $\operatorname{HMf}(\alpha)=f \circ \alpha$, for all $\alpha \in H M X$. It was shown in [Ra] that the map $H M f:\left(H M X, \mathcal{U}_{H M X}\right) \rightarrow\left(H M Y, \mathcal{U}_{H M Y}\right)$ is uniformly continuous. Hence there exists the continuous map $H f: H X \rightarrow H Y$ such that $H f \mid H M X=H M f$. It is easy to see that $H: \mathcal{C} o m p \rightarrow \mathcal{C} o m p$ is a covariant functor and $H M_{n}$ is a subfunctor of $H$ for each $n \in \mathbb{N}$.

Let us remark that the family of functions $S_{H M}(X)$ embed $H M X$ in the product of closed intervals $\prod_{\varphi_{(a, b)} \in S_{H M}(X)} I_{\varphi_{(a, b)}}$ where $I_{\varphi_{(a, b)}}=\left[\min _{x \in X}|\varphi(x)|, \max _{x \in X}\right.$ $|\varphi(x)|]$. Thus, the space $H X$ is the closure of the image of $H M X$. We denote by 
$p_{\varphi_{(a, b)}}: H X \rightarrow I_{\varphi_{(a, b)}}$ the restriction of the natural projection. Let us remark that the function $H f$ could be defined by the condition $p_{\varphi_{(a, b)}} \circ H f=p_{(\varphi \circ f)_{(a, b)}}$ for each $\varphi_{(a, b)} \in S_{H M}(Y)$.

It is shown in $[\mathrm{RR}]$ that $H X$ is a convex subset of $\prod_{\varphi_{(a, b)} \in S_{H M}(X)} I_{\varphi_{(a, b)}}$.

Define the map $e_{1}: H M X \times H M X \times I \rightarrow H M X$ by the condition that $e_{1}\left(\alpha_{1}, \alpha_{2}, t\right)(l)$ is equal to $\alpha_{1}(l)$ if $l<t$ and $\alpha_{2}(l)$ in the opposite case for $\alpha_{1}, \alpha_{2} \in$ $H M X, t \in I$ and $l \in[0,1)$. We consider $H M X$ with the uniformity $\mathcal{U}_{H M X}$ and $I$ with the natural metric. The map $e_{1}: H M X \times H M X \times I \rightarrow H M X$ is uniformly continuous $[\mathrm{RR}]$.

Hence there exists the extension of $e_{1}$ to the continuous map $e: H X \times H X \times I \rightarrow$ $H X$. It is easy to check that $e(\alpha, \alpha, t)=\alpha$, for each $\alpha \in H X$.

We recall that $P X$ is the space of all nonnegative functionals $\mu: C(X) \rightarrow \mathbb{R}$ with norm 1 , and taken in the weak* topology for a compactum $X$ (see [TZ] or [FZ] for more details). Recall that the base of the weak* topology in $P X$ consists of the sets of the form $O\left(\mu_{0}, f_{1}, \ldots, f_{n}, \varepsilon\right)=\left\{\mu \in P X|| \mu\left(f_{i}\right)-\mu_{0}\left(f_{i}\right) \mid<\varepsilon\right.$ for every $1 \leq i \leq n\}$. Hence we can consider $P X$ as a subspace of the product of closed intervals $\prod_{\varphi \in C(X)} I_{\varphi}$ where $I_{\varphi}=\left[\min _{x \in X}|\varphi(x)|, \max _{x \in X}|\varphi(x)|\right]$. We denote by $\pi_{\varphi}: P X \rightarrow I_{\varphi}$ the restriction of the natural projection.

For each $(a, b) \subset(0,1)$ we can define a map $r X_{(a, b)}: H X \rightarrow P X$ by formula $\pi_{\varphi} \circ r X_{(a, b)}=p_{\varphi_{(a, b)}}$. It is easy to check that $r X_{(a, b)}$ is well defined, continuous and affine map.

We define as well a map $i X: P X \rightarrow H X$ by the formula $p_{\varphi_{(a, b)}} \circ i X=\pi_{\varphi}$. We have that $r X_{(a, b)} \circ i X=\operatorname{id}_{P X}$, hence $r X_{(a, b)}$ is a retraction for each $(a, b) \subset(0,1)$. The map $r X_{(0,1)}$ we denote simply by $r X$.

Let us remark that $r_{(a, b)}: H \rightarrow P$ is a natural transformation. (It means that for each map $f: X \rightarrow Y$ we have $\operatorname{Pf\circ } \circ X_{(a, b)}=r Y_{(a, b)} \circ H f$.) The same property is valid for $i: P \rightarrow H$.

\section{Openess of the functor $H$}

A subset $A \subset H X$ is called $e$-convex if $e(\alpha, \beta, t) \in A$, for each $\alpha, \beta \in A$ and $t \in I$. If, additionally, $A$ is convex, we call $A H$-convex.

We suppose that $f: X \rightarrow Y$ is a continuous surjective map between compacta during this section. The proofs of the next three lemmas are easy checking on $H M X$ which is a dense subset of $H X$.

Lemma 3.1. For each $\mu, \nu \in H X$ and $t \in[0,1]$ we have $e(H f(\mu), H f(\nu), t)=$ $H f(e(\mu, \nu, t))$.

Lemma 3.2. Consider any $\nu \in H X$ and $a, b, c \in \mathbb{R}$ such that $0 \leq a<c<b \leq 1$. Then we have $p_{\varphi_{(a, b)}}(\nu)=\frac{c-a}{b-a} p_{\varphi_{(a, c)}}(\nu)+\frac{b-c}{b-a} p_{\varphi_{(c, b)}}(\nu)$ for each $\nu \in H X$.

Lemma 3.3. Let $t \in(0,1)$ and $(a, b) \subset(0,1)$. For each $\mu, \nu \in H X$ and $\varphi \in C(X)$ we have $p_{\varphi_{(a, b)}}(e(\mu, \nu, t))=p_{\varphi_{(a, b)}}(\mu)$ if $b \leq t$ and $p_{\varphi_{(a, b)}}(e(\mu, \nu, t))=p_{\varphi_{(a, b)}}(\nu)$ if $t \leq a$.

Lemma 3.4. Let $A$ be a closed $H$-convex subset of $H X$ and $\nu \notin A$. Then there exist $\varphi \in C(X)$ and $(a, b) \subset(0,1)$ such that. $p_{\varphi_{(a, b)}}(\nu)<p_{\varphi_{(a, b)}}(\mu)$ for each $\mu \in A$.

Proof. Suppose the contrary. We can for each $\mu \in A$ choose $\psi_{\mu} \in S_{H M}(X)$ such that $p_{\psi_{\mu}}(\nu)<p_{\psi_{\mu}}(\mu)$. Since $A$ is compact, there exist $\mu_{1}, \ldots, \mu_{n} \in A$ such that for each $\mu \in A$ there exists $i \in\{1, \ldots, n\}$ such that $p_{\psi_{\mu_{i}}}(\nu)<p_{\psi_{\mu_{i}}}(\mu)$. By Lemma 3.2 we can choose a family of intervals $\left\{\left(a_{i}, b_{i}\right)\right\}_{i=1}^{k}$ such that $b_{i} \leq a_{i+1}$ and for 
each $i \in\{1, \ldots, k\}$ a family of function $\varphi_{\left(a_{i}, b_{i}\right)}^{1}, \ldots, \varphi_{\left(a_{i}, b_{i}\right)}^{n_{i}} \in S_{H M}(X)$ such that for each $\mu \in A$ there exist $i \in\{1, \ldots, k\}$ and $l \in\left\{1, \ldots, n_{i}\right\}$ such that $p_{\varphi_{\left(a_{i}, b_{i}\right)}^{l}}(\nu)<$ $p_{\varphi_{\left(a_{i}, b_{i}\right)}^{l}}(\mu)$.

Consider the set $K=\left\{\mu \in A \mid p_{\varphi_{\left(a_{i}, b_{i}\right)}^{l}}(\mu) \leq p_{\varphi_{\left(a_{i}, b_{i}\right)}^{l}}(\nu)\right.$ for each $i \in\{2, \ldots, k\}$ and $\left.l \in\left\{1, \ldots, n_{i}\right\}\right\}$. Then $K$ is a compact convex subset of $A$ and for each $\mu \in A$ there exists $l \in\left\{1, \ldots, n_{1}\right\}$ such that $p_{\varphi_{\left(a_{1}, b_{1}\right)}^{l}}(\nu)<p_{\varphi_{\left(a_{1}, b_{1}\right)}^{l}}(\mu)$. Then $r X_{\left(a_{1}, b_{1}\right)}(K)$ is a convex compact subset of $P X$ which doesn't contain $r X_{\left(a_{1}, b_{1}\right)}(\nu)$. Then there exists $\psi^{1} \in C(X)$ such that $\pi_{\psi^{1}}\left(r X_{\left(a_{1}, b_{1}\right)}(\nu)\right)<\pi_{\psi^{1}}(\eta)$ for each $\eta \in r X_{\left(a_{1}, b_{1}\right)}(K)$. Hence, for each $\mu \in K$ we have $p_{\psi_{\left(a_{1}, b_{1}\right)}^{1}}(\nu)<p_{\psi_{\left(a_{1}, b_{1}\right)}^{1}}(\mu)$.

Proceeding, we obtain $\psi^{1}, \ldots, \psi^{k} \in C(X)$ such that for each $\mu \in A$ there exists $i \in\{1, \ldots, k\}$ such that $p_{\psi_{\left(a_{i}, b_{i}\right)}^{i}}(\nu)<p_{\psi_{\left(a_{i}, b_{i}\right)}^{i}}(\mu)$.

By our supposition we can choose $\mu_{i} \in A$ for each $i \in\{1, \ldots, k\}$ such that $p_{\psi_{\left(a_{i}, b_{i}\right)}^{i}}\left(\mu_{i}\right) \leq p_{\psi_{\left(a_{i}, b_{i}\right)}^{i}}(\nu)$. Put $\xi_{1}=\mu_{1}$ and $\xi_{i+1}=e\left(\xi_{i}, \mu_{i+1}, b_{i}\right)$ for $i \in\{1, \ldots, k-$ $1\}$. Since $A$ is $e$-convex, $\xi_{k} \in A$. By Lemma 3.3 we have $p_{\psi_{\left(a_{i}, b_{i}\right)}^{i}}\left(\xi_{k}\right) \leq p_{\psi_{\left(a_{i}, b_{i}\right)}^{i}}(\nu)$ for each $i \in\{1, \ldots, k\}$. Thus, we obtain a contradiction and the lemma is proved.

The proof of the next lemma follows from Lemma 3.1 and the fact that $H f$ is affine map.

Lemma 3.5. $(H f)^{-1}(\nu)$ is $H$-convex for each $\nu \in H Y$.

Let $f: X \rightarrow Y$ be a map and $\varphi \in C(X)$. By $\varphi_{*}$ we denote the function $\varphi_{*}: Y \rightarrow \mathbb{R}$ defined by the formula $\varphi_{*}(y)=\inf \left(\varphi\left(f^{-1}(y)\right), y \in Y\right.$. It is known [DE] that if $f$ is open then the function $\varphi_{*}$ is continuous.

Proof of Theorem 1.1. Let $f: X \rightarrow Y$ be a map such that the map $H f$ : $H X \rightarrow H Y$ is open. Let us show that the map $P f$ is open. Consider any open set $U \subset P X$ and $\mu \in U$. Then $(r X)^{-1}(U)$ is an open set in $H X$ and $i X(\mu) \in(r X)^{-1}(U)$ Since $H f$ is an open map, $H f\left((r X)^{-1}(U)\right)$ is open in $H Y$ and $H f(i X(\mu)) \in H f\left((r X)^{-1}(U)\right)$. Since $r$ is a natural transformation, we have $H f\left((r X)^{-1}(U)\right) \subset(r Y)^{-1}(P f(U))$. We have $i Y(P f(\mu))=H f(i X(\mu))$ or $P f(\mu) \in$ $(i Y)^{-1}\left(H f\left((r X)^{-1}(U)\right)\right) \subset(i Y)^{-1}\left((r Y)^{-1}(\operatorname{Pf}(U))\right)=\operatorname{Pf}(U)$. Since $(i Y)^{-1}\left(H f\left((r X)^{-1}(U)\right)\right)$ is open, the map $P f$ is open. Hence $f$ is open as well $[\mathrm{DE}]$.

Now let a map $f: X \rightarrow Y$ be open. Let us suppose that $H f$ is not open. Then there exists $\mu_{0} \in H X$, a net $\left\{\nu_{\alpha}, \alpha \in \mathcal{A}\right\} \subset O(Y)$ converging to $\nu_{0}=\operatorname{Hf}\left(\mu_{0}\right)$ and a neighborhood $W$ of $\mu_{0}$ such that $(H f)^{-1}\left(\nu_{\alpha}\right) \cap W=\emptyset$ for each $\alpha \in \mathcal{A}$. Since $H M(Y)$ is a dense subset of $H Y$, we can suppose that all $\nu_{\alpha} \in H M(Y)$. Since $H X$ is a compactum, we can assume that the net $A_{\alpha}=(H f)^{-1}\left(\nu_{\alpha}\right)$ converges in $\exp (H X)$ to some closed subset $A \subset H X$. It is easy to check that $A \subset(H f)^{-1}\left(\nu_{0}\right)$ and $\mu_{0} \notin A$. By the Lemma 3.5 all the sets $A_{\alpha}$ are $H$-convex. It is easy to see that $A$ is $H$-convex as well. Since $\mu_{0} \notin A$, there exists by Lemma $3.4 \varphi \in C(X)$ and $(a, b) \subset(0,1)$ such that $p_{\varphi_{(a, b)}}\left(\mu_{0}\right)<p_{\varphi_{(a, b)}}(\mu)$ for each $\mu \in A$. Consider any $\alpha \in \mathcal{A}$. Let $\left\{y_{1}, \ldots, y_{s}\right\}=\nu_{\alpha}([0,1))$. Choose for each $y_{i}$ the point $x_{i}$ such that $f\left(x_{i}\right)=y_{i}$ and $\varphi\left(x_{i}\right)=\varphi_{*}\left(y_{i}\right)$. Define a map $j:\left\{y_{1}, \ldots, y_{s}\right\} \rightarrow\left\{x_{1}, \ldots, x_{s}\right\}$ by the formula $j\left(y_{i}\right)=x_{i}$ and put $\mu_{\alpha}(t)=j \circ \nu_{\alpha}(t)$ for $t \in[0,1)$. Let $\mu$ be a limit point of the net $\mu_{\alpha}$, then $\mu \in A$. Since $\varphi_{(a, b)}\left(\mu_{\alpha}\right)=\varphi_{*(a, b)}\left(\nu_{\alpha}\right)$, we have $p_{\varphi_{(a, b)}}(\mu)=p_{\varphi_{*(a, b)}}\left(\nu_{0}\right)=p_{\left(\varphi_{*} \circ f\right)_{(a, b)}}\left(\mu_{0}\right) \leq p_{\varphi_{(a, b)}}\left(\mu_{0}\right)$. We have obtained the contradiction and the theorem is proved. 


\section{Proofs}

We will need some notations and facts from the theory of non-metrizable compacta. See [10] for more details.

Let $\tau$ be an infinite cardinal number. A partially ordered set $\mathcal{A}$ is called $\tau$ complete, if every subset of cardinality $\leq \tau$ has a least upper bound in $\mathcal{A}$. An inverse system consisting of compacta and surjective bonding maps over a $\tau$-complete indexing set is called $\tau$-complete. A continuous $\tau$-complete system consisting of compacta of weight $\leq \tau$ is called a $\tau$-system.

As usual, by $\omega$ we denote the countable cardinal number.

A compactum $X$ is called openly generated if $X$ can be represented as the limit of an $\omega$-system with open bonding maps.

Proof of Theorem 1.2. It was shown in [RR] that $H X$ is an absolute retract for each metrizable compactum $X$. So, we can consider only non-metrizable case.

Let $X$ be an openly generated compactum of weight $\leq \omega_{1}$. By Theorem 1.1 the compactum $H X$ is also openly generated. Since weight of $X$ (and $H X$ [Ra]) is $\leq \omega_{1}, H X$ is $A E(0)$. Since $H X$ is a convex compactum, $H X$ is $A R[\mathrm{Fe}]$.

Now, suppose $H X \in A R$. Since $r X: H X \rightarrow P X$ ia a retraction, $P X$ is an $A R$ too. Then $X$ is an openly generated compactum of weight $\leq \omega_{1}[\mathrm{Fe}]$. The theorem is proved.

By $w(X)$ we denote the weight of the compactum $X$, by $\chi(x, X)$ the character in the point $x$ and by $\chi(X)$ the character of the space $X$. The space $X$ is called $\chi$-homogeneous if for each $x, y \in X$ we have $\chi(x, X)=\chi(y, X)$. We will use the following characterization of the Tychonov cube $I^{\tau}$. An $A R$-compactum $X$ of weight $\tau$ is homeomorphic to $I^{\tau}$ for an uncountable cardinal number $\tau$ if and only if $X$ is $\chi$-homogeneous [Sh1].

Let $x \in X$. Define $\delta(x) \in H X$ by the condition $p_{\varphi_{(a, b)}}(\delta(x))=\varphi(x)$ for each $\varphi_{(a, b)} \in S_{H M}(X)$.

Lemma 4.1. Let $f: X \rightarrow Y$ be an open map. Then $H f$ has a degenerate fiber if and only if $f$ has a degenerate fiber.

Proof. Let $f: X \rightarrow Y$ be an open map such that there exists $y \in Y$ with $f^{-1}(y)=$ $\{x\}, x \in X$. Consider any $\mu \in H X$ with $H f(\mu)=\delta(y)$. Let us show that $\mu=\delta(x)$. Consider any $\varphi_{(a, b)} \in S_{H M}(X)$. Suppose that $p_{\varphi_{(a, b)}}(\mu) \neq \varphi(x)$. We can assume that $p_{\varphi_{(a, b)}}(\mu)<\varphi(x)$. By [Ra, Lemma 1] there exists a function $\psi \in C(Y)$ such that $\psi(y)=\varphi(x)$ and $\psi \circ f \leq \varphi$. Then we have $p_{(\psi \circ f)_{(a, b)}}(\mu) \leq p_{\varphi_{(a, b)}}(\mu)<\varphi(x)$ and $p_{\psi_{(a, b)}}(\delta(y))=p_{\psi_{(a, b)}} O(f)(\mu)=p_{(\psi \circ f)_{(a, b)}}(\mu)<\varphi(x)=\psi(y)$. Hence we obtain the contradiction. Thus, $H f$ has a degenerate fiber.

Now, suppose $f$ has no degenerate fiber. Consider any $\mu \in H Y$. Take any $y \in \operatorname{supp} \mu \subset Y$. Since $f$ is an open map and $f^{-1}(y)$ is not a singleton, we can choose two closed subsets $A_{1}, A_{2} \subset X$ such that $f\left(A_{1}\right)=f\left(A_{2}\right)=Y$ and $\left(A_{1} \cap f^{-1}(y)\right) \cap\left(A_{2} \cap f^{-1}(y)\right)=\emptyset$. Since the functor $H$ preserves surjective maps [Ra], there exist $\mu_{1} \in H\left(A_{1}\right)$ and $\mu_{2} \in H\left(A_{2}\right)$ such that $\operatorname{Hf}\left(\mu_{1}\right)=H f\left(\mu_{2}\right)=\mu$. Since $y \in \operatorname{supp} \mu$, there exist $y_{1} \in \operatorname{supp} \mu_{1} \subset A_{1}$ and $y_{2} \in \operatorname{supp} \mu_{2} \subset A_{2}$ such that $f\left(y_{1}\right)=f\left(y_{2}\right)=y$. Hence $\mu_{1} \neq \mu_{2}$ and the lemma is proved.

Lemma 4.2. An openly generated compactum $X$ of weight $\omega_{1}$ is $\chi$-homogeneous if and only if $H X$ is $\chi$-homogeneous.

Proof. Let $H X$ be $\chi$-homogeneous. Since the functor $H$ preserves the weight [Ra], $H X$ is an absolute retract such that $\chi(\mu, H X)=\omega_{1}$ for each $\mu \in H X$. Then take 
any $x \in X$ and suppose that there exists $\left\{U_{i} \mid i \in \mathbb{N}\right\}$ a countable base of open neighborhoods. Consider a family of functions $\left\{\varphi_{i} \in C(X) \mid i \in \mathbb{N}\right\}$ such that $\varphi_{i}(x)=1, \varphi_{i} \mid X \backslash U_{\subset}=0$. Then the family of function $\left\{\varphi_{i(a, b)} \mid i \in \mathbb{N} ; a, b \in \mathbb{Q}\right\}$ define a countable base of neighborhoods of $\delta(x)$ in $H X$. We obtain a contradiction, hence $X$ is $\chi$-homogeneous of $X$.

Now let $X$ be a $\chi$-homogeneous openly generated compactum of weight $\omega_{1}$. Then $\chi(X)=\omega_{1}[\mathrm{Ra}$, Lemma 4]. Suppose that there exists a point $\nu \in H X$ such that $\chi(\nu, H X)<\omega_{1}$. Represent $X$ as the limit space of an $\omega$-system $\left\{X_{\alpha}, p_{\alpha}, \mathcal{A}\right\}$ with open limit projections $p_{\alpha}$. There exists $\alpha \in \mathcal{A}$ such that $\left(H p_{\alpha}\right)^{-1}\left(H p_{\alpha}(\nu)\right)=\{\nu\}$. By Lemma 4.2 there exists a point $z \in X_{\alpha}$ such that $p_{\alpha}^{-1}(z)=\{x\}, x \in X$. Hence $\chi(x, X)<\omega_{1}$ and we obtain the contradiction. The lemma is proved.

Proof of Theorem 1.3. The proof of the theorem follows from Theorem 1.2 and Lemma 4.2 .

\section{REFERENCES}

[DE] S. Ditor and L. Eifler, Some open mapping theorems for measures, Trans. Amer. Math. Soc. 164 (1972), 287-293.

[Fe] V. V. Fedorchuk, Probability measures in topology, Uspekhi Mat. Nauk 46 (1991), 41-80. (Russian)

[FZ] V. V. Fedorchuk and M. M. Zarichnyi, Covariant functors in categories of topological spaces, Results of Science and Technology, Algebra.Topology.Geometry 28, VINITI, Moscow, pp. 47-95. (Russian)

[HM] S. Hartman and J. Mycielski, On the embedding of topological groups into connected topological groups, Colloq. Math. 5 (1958), 167-169.

[Ra] T. Radul, A normal functor based on the Hartman-Mycielski construction, Mat. Studii 19 (2003), 201-207.

[RR] T. Radul and D. Repovš, On topological properties of the Hartman-Mycielski functor, Proc. Indian Acad. Sci. Math. Sci. 115 (2005), 477-482.

[Sh1] E. V. Shchepin, On Tychonov manifolds, Dokl.Akad.Nauk USSR 246 (1979), 551-554. (Russian)

[Sh2] E. V. Shchepin, Functors and uncountable powers of compacta, Uspekhi Mat. Nauk 36 (1981), 3-62. (Russian)

[TZ] A. Telejko and M. Zarichnyi, Categorical Topology of Compact Hausdorff Spaces, Lviv, VNTL, 1999, p. 263.

Department of Mechanics and Mathematics, Lviv National University, UniverSYTETSKa ST.,1, 79602 LVIV, UKRAINE.

E-mail address: tarasradul@yahoo.co.uk

Institute For Matematics, Physics and Mechanics, and University Of Ljubljana, P.O.Box 2964, Luubluana, Slovenia 1001

E-mail address: dusan.repovs@guest.arnes.si 